\title{
Multiple soliton solutions for the variant Boussinesq equations
}

\author{
Peng Guo ${ }^{1,2,3}$, Xiang Wu $\mathrm{W}^{2,3}$ and Liang-bi Wang ${ }^{2,3}$
}

"Correspondence:

guopenglzjtu@126.com

'School of Mathematics and

Physics, Lanzhou Jiaotong

University, Lanzhou, 730070,

P.R. China

${ }^{2}$ School of Mechatronic

Engineering, Lanzhou Jiaotong

University, Lanzhou, 730070,

P.R. China

Full list of author information is

available at the end of the article

\begin{abstract}
The Hirota bilinear method is used to handle the variant Boussinesq equations. Multiple soliton solutions and multiple singular soliton solutions are formally established. It is shown that the Hirota bilinear method may provide us with a straightforward and effective mathematic tool for generating multiple soliton solutions of nonlinear wave equations in fluid mechanics.
\end{abstract}

Keywords: Hirota bilinear method; the variant Boussinesq equations; multiple soliton solutions; multiple singular soliton solutions

\section{Introduction}

Many phenomena in physics, biology, chemistry, mechanics, etc. are described by nonlinear partial differential equations. Nonlinear wave phenomena of dissipation, diffusion, reaction, and convection are very important, and they can be represented with a variety of nonlinear wave equations. The investigation of exact solutions of these equations will help ones to understand these phenomena better.

During the past several decades, many powerful and efficient methods have been proposed to obtain the exact solutions of nonlinear wave equations, such as inverse scattering method [1], Darboux and Bäcklund transformations [2, 3], the Hirota bilinear method [4], the tanh method [5], the extended tanh method [6], the sine-cosine method [7], the homogeneous balance method [8], the homotopy perturbation method $[9,10]$, the $F$-expansion method [11], the Exp-function expansion method [12, 13], the $\left(G^{\prime} / G\right)$-expansion method $[14,15]$, the Kudryashov method [16], the mapping method [17], the extended mapping method [18], and so on.

The above methods can be used to handle the nonlinear wave equations for single soliton solutions, but the multiple soliton solutions of the nonlinear wave equations can be obtained only by three different methods: the inverse scattering method, the Bäcklund transformation method, and the Hirota bilinear method. However, the Hirota bilinear method is rather heuristic and possesses significant features that make it be ideal for the determination of multiple soliton solutions for a wide class of the nonlinear wave equations [19-25]. When the Hirota bilinear method is used, computer symbolic systems such as Maple and Mathematica allow us to perform complicated and tedious calculations.

The Boussinesq equation is a well-known model of long water wave of moderate amplitude, describes one dimensional, weakly nonlinear internal wave which develops at the boundary between two immiscible fluids. In addition, the equation is a simplified

() 2015 Guo et al.; licensee Springer. This is an Open Access article distributed under the terms of the Creative Commons Attribution License (http://creativecommons.org/licenses/by/4.0), which permits unrestricted use, distribution, and reproduction in any medium, provided the original work is properly credited. 
model of the atmospheric movement equation which is applicable to mesoscale and quasiincompressible fluid movement, which means important physical applications in hydrodynamics. The Boussinesq equation also is of considerable mathematic interests because of its rich mathematical structures. In the present research, we focus on the variant Boussinesq equations, which was derived by Sachs [26] in 1988 as a model for water waves:

$$
\left\{\begin{array}{l}
H_{t}+(H u)_{x}+u_{x x x}=0 \\
u_{t}+H_{x}+u u_{x}=0
\end{array}\right.
$$

where $u(x, t)$ is the velocity, $H(x, t)$ is the height of free wave surface for fluid in the trough, and the subscripts denote partial derivatives. In the past years, many authors have studied Eq. (1). For example, Wang [27] solved Eq. (1) by the homogeneous balance method. Yan and Zhang [28] obtained new explicit and exact traveling wave solutions for Eq. (1) by an improved sine-cosine method and the Wu elimination method. Naz et al. [29] obtained the conservation laws for Eq. (1) by an interesting method of increasing the order of partial differential equations. Fan and Hon [30] uniformly constructed a series of traveling wave solutions for Eq. (1) by a new algebraic method. Lü [31] solved Eq. (1) by a general Jacobi elliptic function expansion method, and obtained Jacobi elliptic function solutions. Yuan et al. [32] constructed bifurcations of traveling wave solutions for Eq. (1) by the bifurcation theory of planar dynamical systems. Li et al. [33] obtained all possible smooth, cusped solitary wave solutions for Eq. (1) by the phase portrait analytical technique.

The above review shows that many works to obtain the exact solutions of Eq. (1) have been carried out in recent years, but the multiple soliton solutions for Eq. (1) have not been obtained. The existence of multiple soliton solutions often implies the integrability of the considered equations. The objectives of this paper are twofold. First, we aim to apply the Hirota bilinear method to handle Eq. (1). Second, we seek to establish multiple soliton solutions and multiple singular soliton solutions to confirm that Eq. (1) is completely integrable. The rest of this paper is organized as follows. In Section 2, the Hirota bilinear method for finding the multiple soliton solutions of the nonlinear wave equations is described. In Sections 3 and 4, the method to solve Eq. (1) is illustrated in detail. Multiple soliton solutions and multiple singular soliton solutions are obtained. In Section 5, some conclusions are given.

\section{The Hirota bilinear method}

The Hirota direct method is well known, and it gives soliton solutions by polynomials of exponentials. We only summarize the main steps as follows.

(i) Substituting

$$
u(x, t)=\mathrm{e}^{\theta_{i}}, \quad \theta_{i}=k_{i} x-c_{i} t
$$

into the linear terms of the equation under discussion to determine the dispersion relation between $k_{i}$ and $c_{i}$.

(ii) Substituting the single soliton solution

$$
u(x, t)=R[\ln f(x, t)]_{x}=R \frac{f_{x}}{f},
$$




$$
u(x, t)=R[\ln f(x, t)]_{x x}=R \frac{f_{x x}-f_{x}^{2}}{f^{2}},
$$

or

$$
u(x, t)=R\left[\arctan \left(\frac{f(x, t)}{g(x, t)}\right)\right]_{x}=R \frac{f_{x} g-f g_{x}}{f^{2}+g^{2}}
$$

into the equation under discussion to determine $R$, where

$$
f(x, t)=1+e^{\theta_{1}} .
$$

(iii) For a single soliton, we use

$$
f(x, t)=1+e^{\theta_{1}} .
$$

(iv) For two soliton solutions, we use

$$
f(x, t)=1+e^{\theta_{1}}+e^{\theta_{2}}+a_{12} e^{\theta_{1}+\theta_{2}} .
$$

(v) For three soliton solutions, we use

$$
f(x, t)=1+e^{\theta_{1}}+e^{\theta_{2}}+e^{\theta_{3}}+a_{12} e^{\theta_{1}+\theta_{2}}+a_{23} e^{\theta_{2}+\theta_{3}}+a_{13} e^{\theta_{1}+\theta_{3}}+a_{123} e^{\theta_{1}+\theta_{2}+\theta_{3}} .
$$

If the obtained result shows that $a_{123}=a_{12} a_{23} a_{13}$, then the equation gives multiple soliton solutions and the equation is integrable.

However, for multiple singular soliton solutions, we apply the following steps:

(i) For the dispersion relation, we use

$$
u(x, t)=\mathrm{e}^{\theta_{i}}, \quad \theta_{i}=k_{i} x-c_{i} t
$$

(ii) Next we substitute the single soliton solution

$$
\begin{aligned}
& u(x, t)=R[\ln f(x, t)]_{x}=R \frac{f_{x}}{f}, \\
& u(x, t)=R[\ln f(x, t)]_{x x}=R \frac{f_{x x}-f_{x}^{2}}{f^{2}},
\end{aligned}
$$

or

$$
u(x, t)=R\left[\arctan \left(\frac{f(x, t)}{g(x, t)}\right)\right]_{x}=R \frac{f_{x} g-f g_{x}}{f^{2}+g^{2}}
$$

into the equation under discussion to determine $R$, where

$$
f(x, t)=1-e^{\theta_{1}} .
$$

(iii) For a single soliton, we use

$$
f(x, t)=1-e^{\theta_{1}} .
$$


(iv) For two soliton solutions, we use

$$
f(x, t)=1-e^{\theta_{1}}-e^{\theta_{2}}+a_{12} e^{\theta_{1}+\theta_{2}} .
$$

(v) For three soliton solutions, we use

$$
f(x, t)=1-e^{\theta_{1}}-e^{\theta_{2}}-e^{\theta_{3}}+a_{12} e^{\theta_{1}+\theta_{2}}+a_{23} e^{\theta_{2}+\theta_{3}}+a_{13} e^{\theta_{1}+\theta_{3}}-a_{123} e^{\theta_{1}+\theta_{2}+\theta_{3}} .
$$

Other approaches will also be used for the multiple singular soliton solutions, as will be examined later.

\section{Multiple soliton solutions of the variant Boussinesq equations}

Substituting the following equations:

$$
\left\{\begin{array}{l}
H(x, t)=A e^{k_{i} x-c_{i} t} \\
u(x, t)=B e^{k_{i} x-c_{i} t}
\end{array}\right.
$$

into the linear terms of Eq. (1), we obtain the dispersion relation

$$
c_{i}= \pm k_{i}^{2}
$$

and as a result we have

$$
\theta_{i}=k_{i} x \mp k_{i}^{2} t
$$

where $A$ and $B$ are constants. Using the Cole-Hopf transformation method, we assume that the multiple solutions of Eq. (1) are

$$
\left\{\begin{array}{l}
H(x, t)=R_{1}[\ln f(x, t)]_{x x}=R_{1} \frac{f_{x x}-f_{x}^{2}}{f^{2}} \\
u(x, t)=R_{2}[\ln f(x, t)]_{x}=R_{2} \frac{f_{x}}{f}
\end{array}\right.
$$

where $f(x, t)$, for the single soliton solution, is given by

$$
f(x, t)=1+e^{\theta_{1}}=1+e^{k_{1} x \mp k_{1}^{2} t} .
$$

Substituting Eq. (22) into Eq. (21), using the result from Eq. (1), and solving it for $R_{1}$ and $R_{2}$, we find

$$
R_{1}=R_{2}=2 \text {. }
$$

Substituting Eqs. (22)-(23) into Eq. (21) we obtain the single soliton solution

$$
\left\{\begin{array}{l}
H(x, t)=\frac{2 k_{1}^{2} e^{k_{1} x+k_{1}^{2} t}}{\left(\mathrm{e}_{1}^{2} t+\mathrm{e}^{k_{1} x}\right)^{2}}, \\
u(x, t)=\frac{2 k_{1} \mathrm{e}^{k_{1} x-k_{1}^{2} t}}{1+\mathrm{e}^{k_{1} x-k_{1}^{2} t}},
\end{array}\right.
$$




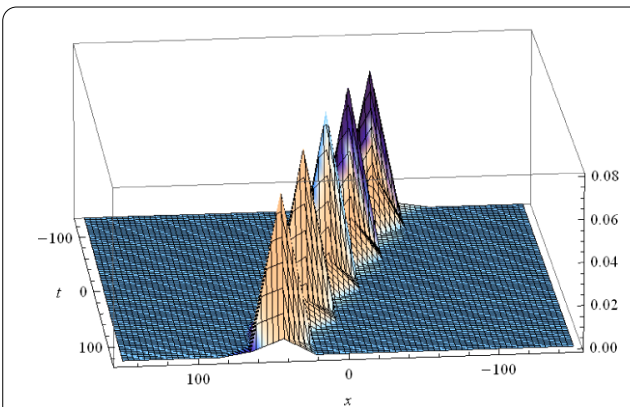

(a)

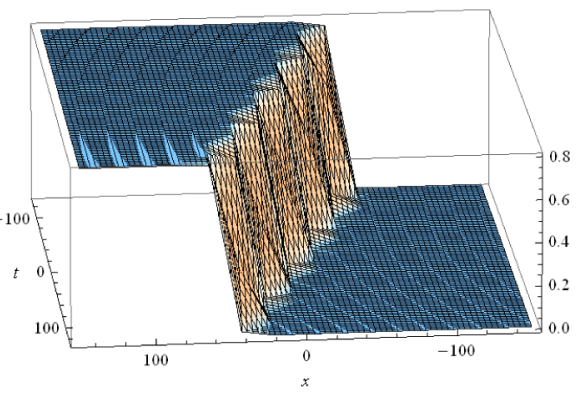

(b)

Figure 1 Plots of the solution described by Eq. (24). (a) The single soliton solution $H(x, t)$ for $k_{1}=0.4$. (b) The single soliton solution $u(x, t)$ for $k_{1}=0.4$

$$
\left\{\begin{array}{l}
H(x, t)=\frac{2 k_{1}^{2} \mathrm{e}^{k_{1} x+k_{1}^{2} t}}{\left(1+\mathrm{e}^{k_{1} x+k_{1}^{2} t}\right)^{2}}, \\
u(x, t)=\frac{2 k_{1} \mathrm{e}^{k_{1} x+k_{1}^{2} t}}{1+\mathrm{e}^{k_{1} x+k_{1}^{2} t}} .
\end{array}\right.
$$

Figure 1 shows the single soliton solution for Eq. (1) for some special values of the solution's parameters in Eq. (24).

For two soliton solutions, we set

$$
f(x, t)=1+e^{\theta_{1}}+e^{\theta_{2}}+a_{12} e^{\theta_{1}+\theta_{2}}=1+e^{k_{1} x \mp k_{1}^{2} t}+e^{k_{2} x \mp k_{2}^{2} t}+a_{12} e^{\left(k_{1}+k_{2}\right) x \mp\left(k_{1}^{2}+k_{2}^{2}\right) t} .
$$

Using Eqs. (23) and (26) into Eq. (21) and substituting the result into Eq. (1), we obtain the phase shift by

$$
a_{12}=0
$$

and hence we set

$$
a_{i j}=0, \quad 1 \leq i<j \leq N .
$$

The two soliton solutions are obtained by substituting Eqs. (26) and (27) into Eq. (21),

$$
\begin{aligned}
& \left\{\begin{array}{l}
H(x, t)=\frac{2 \mathrm{e}^{\left(k_{1}^{2}+k_{2}^{2}\right) t}\left[k_{1}^{2} \mathrm{e}^{k_{1} x+k_{2}^{2} t}+\left(k_{1}-k_{2}\right)^{2} \mathrm{e}^{\left(k_{1}+k_{2}\right) x}+k_{2}^{2} \mathrm{e}^{k_{2} x+k_{1}^{2} t}\right]}{\left(\mathrm{e}_{1}^{\left(k_{1}^{2}+k_{2}^{2}\right) t}+\mathrm{e}^{k_{1} x+k_{2}^{2} t}+\mathrm{e}^{k_{2} x+k_{1}^{2} t}\right)^{2}}, \\
u(x, t)=\frac{2\left(k_{1} \mathrm{e}^{k_{1} x-k_{1}^{2} t}+k_{2} \mathrm{e}^{\left.k_{2} x-k_{2}^{2} t\right)}\right.}{1+\mathrm{e}^{k_{1} x-k_{1}^{2} t}+\mathrm{e}^{k_{2} x-k_{2}^{2} t}},
\end{array}\right. \\
& \left\{\begin{array}{l}
H(x, t)=\frac{2 k_{1}^{2} \mathrm{e}^{k_{1} x+k_{1}^{2} t}+2 \mathrm{e}^{k_{2} x+k_{2}^{2} t}\left[\left(k_{1}-k_{2}\right)^{2} \mathrm{e}^{k_{1} x+k_{1}^{2} t}+k_{2}^{2}\right]}{\left(1+\mathrm{k}_{1} x+k_{1}^{2} t+\mathrm{e}^{k_{2} x+k_{2}^{2} t}\right)^{2}}, \\
u(x, t)=\frac{2\left(k_{1} \mathrm{e}^{k_{1} x+k_{1}^{2} t}+\mathrm{k}_{2} \mathrm{e}^{k_{2} x+k_{2}^{2} t}\right)}{1+\mathrm{e}^{k_{1} x+k_{1}^{2} t}+\mathrm{e}^{k_{2} x+k_{2}^{2} t}} .
\end{array}\right.
\end{aligned}
$$

Figure 2 shows the two soliton solutions for Eq. (1) for some special values of the solution's parameters in Eq. (29).

For three soliton solutions, we set

$$
f(x, t)=1+e^{\theta_{1}}+e^{\theta_{2}}+e^{\theta_{3}}=1+e^{k_{1} x \mp k_{1}^{2} t}+e^{k_{2} x \mp k_{2}^{2} t}+e^{k_{3} x \mp k_{3}^{2} t} .
$$




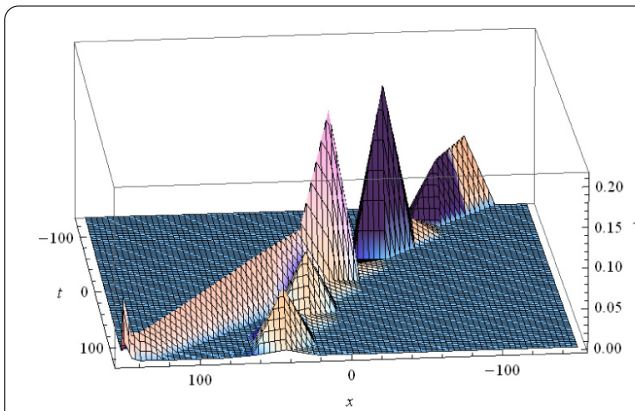

(a)

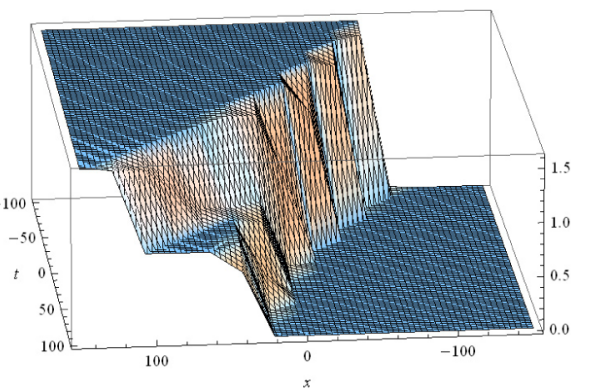

(b)

Figure 2 Plots of the solution described by Eq. (29). (a) The two soliton solutions $H(x, t)$ for $k_{1}=0.4$, $k_{2}=0.8$. (b) The two soliton solutions $u(x, t)$ for $k_{1}=0.4, k_{2}=0.8$.

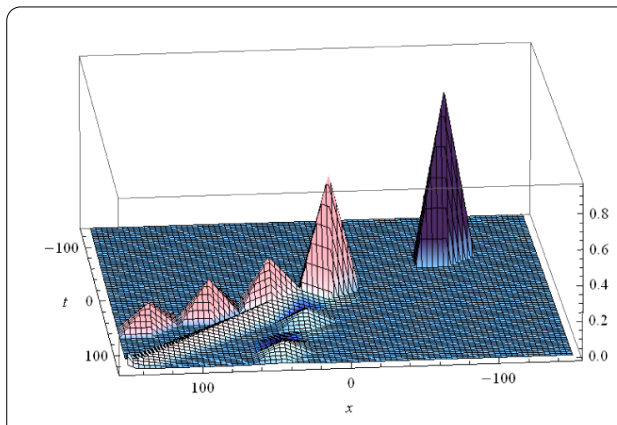

(a)

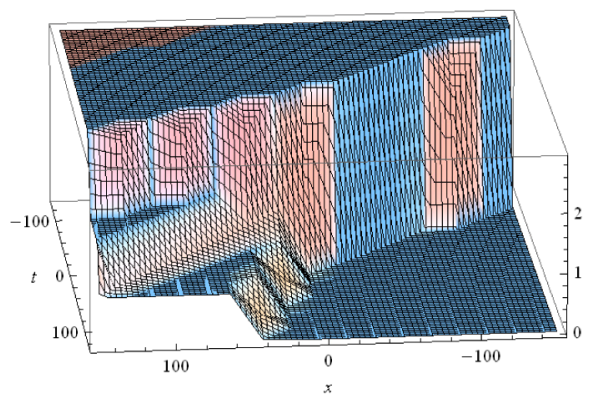

(b)

Figure 3 Plots of the solution described by Eq. (32). (a) The three soliton solutions $H(x, t)$ for $k_{1}=0.4$, $k_{2}=0.8, k_{3}=1.55$. (b) The three soliton solutions $u(x, t)$ for $k_{1}=0.4, k_{2}=0.8, k_{3}=1.45$.

Proceeding as before, we find that the three soliton solutions are given by

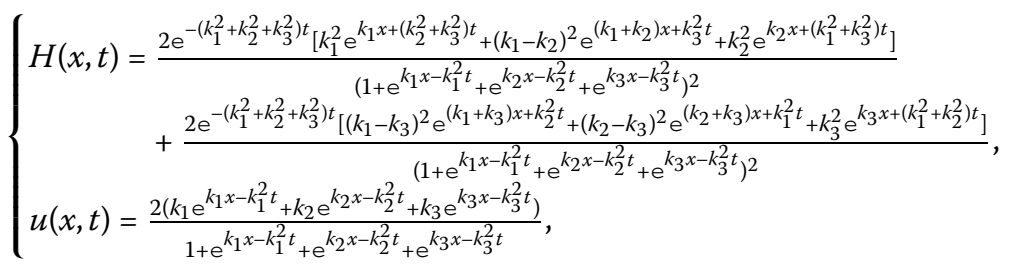

$$
\begin{aligned}
& \int H(x, t)=\frac{2\left[k_{1}^{2} \mathrm{e}^{k_{1} x+k_{1}^{2} t}+\left(k_{1}-k_{2}\right)^{2} \mathrm{e}^{\left(k_{1}+k_{2}\right) x+\left(k_{1}^{2}+k_{2}^{2}\right) t}+k_{2}^{2} \mathrm{e}^{k_{2} x+k_{2}^{2} t}\right]}{\left(1+\mathrm{e}^{k_{1} x+k_{1}^{2} t}+\mathrm{e}^{k_{2} x+k_{2}^{2} t}+\mathrm{e}^{k_{3} x+k_{3}^{2} t}\right)^{2}} \\
& +\frac{2\left[\left(k_{1}-k_{3}\right)^{2} \mathrm{e}^{\left(k_{1}+k_{3}\right) x+\left(k_{1}^{2}+k_{3}^{2}\right) t}+\left(k_{2}-k_{3}\right)^{2} \mathrm{e}^{\left(k_{2}+k_{3}\right) x+\left(k_{2}^{2}+k_{3}^{2}\right) t}+k_{3}^{2} \mathrm{e}^{k_{3} x+k_{3}^{2} t}\right]}{\left(1+\mathrm{e}^{k_{1} x+k_{1}^{2} t}+\mathrm{e}^{k_{2} x+k_{2}^{2} t}+\mathrm{e}^{k_{3} x+k_{3}^{2} t}\right)^{2}}, \\
& u(x, t)=\frac{2\left(k_{1} e^{k_{1} x+k_{1}^{2} t}+k_{2} e^{k_{2} x+k_{2}^{2} t}+k_{3} \mathrm{e}^{k_{3} x+k_{3}^{2} t}\right)}{1+\mathrm{e}^{k_{1} x+k_{1}^{2} t}+\mathrm{e}^{k_{2} x+k_{2}^{2} t}+\mathrm{e}^{k_{3} x+k_{3}^{2} t}} .
\end{aligned}
$$

Figure 3 shows the three soliton solutions for Eq. (1) for some special values of the solution's parameters in Eq. (32).

The three soliton solutions are obtained by substituting Eq. (31) into Eq. (21). This shows that Eq. (1) is completely integrable and $N$ soliton solutions can be determined for $H(x, t)$ and $u(x, t)$, for finite $N$, where $N \geq 1$. Based on Eqs. (32) and (33), the general soliton 
solutions can be set as

$$
\begin{aligned}
& \left\{\begin{array}{l}
H(x, t)=\frac{2\left[\sum_{i=1}^{N} k_{i}^{2} \mathrm{e}^{k_{i} x-k_{i}^{2} t}+\sum_{1 \leq i<j \leq N}\left(k_{i}-k_{j}\right)^{2} \mathrm{e}^{\left(k_{i}+k_{j}\right) x-\left(k_{i}^{2}+k_{j}^{2}\right) t}\right]}{\left(1+\sum_{i=1}^{N} \mathrm{e}^{k_{i} x-k_{i}^{2} t}\right)^{2}}, \\
u(x, t)=\frac{2 \sum_{i=1}^{N} k_{i} \mathrm{e}^{k_{i} x-k_{i}^{2} t}}{1+\sum_{i=1}^{N} \mathrm{e}^{k_{i} x-k_{i}^{2} t}},
\end{array}\right. \\
& \left\{\begin{array}{l}
H(x, t)=\frac{2\left[\sum_{i=1}^{N} k_{i}^{2} \mathrm{e}^{k_{i} x+k_{i}^{2} t}+\sum_{1 \leq i<j \leq N}\left(k_{i}-k_{j}\right)^{2} \mathrm{e}^{\left(k_{i}+k_{j}\right) x+\left(k_{i}^{2}+k_{j}^{2}\right) t}\right]}{\left(1+\sum_{i=1}^{N} \mathrm{e}^{k_{i} x+k_{i}^{2} t}\right)^{2}}, \\
u(x, t)=\frac{2 \sum_{i=1}^{N} k_{i} \mathrm{e}_{i} x+k_{i}^{2} t}{1+\sum_{i=1}^{N} \mathrm{e}^{k_{i} x+k_{i}^{2} t}} .
\end{array}\right.
\end{aligned}
$$

\section{Multiple singular soliton solutions of the variant Boussinesq equations}

Substituting

$$
\left\{\begin{array}{l}
H(x, t)=A e^{k_{i} x-c_{i} t}, \\
u(x, t)=B \mathrm{e}^{k_{i} x-c_{i} t},
\end{array}\right.
$$

into the linear terms of Eq. (1) to find that the dispersion relation is

$$
c_{i}= \pm k_{i}^{2}
$$

and as a result we obtain

$$
\theta_{i}=k_{i} x \mp k_{i}^{2} t,
$$

where $A$ and $B$ are constants. Using the Cole-Hopf transformation method, the multiple singular soliton solutions of Eq. (1) are assumed to be

$$
\left\{\begin{array}{l}
H(x, t)=R_{1}[\ln f(x, t)]_{x x}=R_{1} \frac{f_{f x x}-f_{x}^{2}}{f^{2}}, \\
u(x, t)=R_{2}[\ln f(x, t)]_{x}=R_{2} \frac{f_{x}}{f}
\end{array}\right.
$$

where $f(x, t)$, for the single singular soliton solution, is given by

$$
f(x, t)=1-e^{\theta_{1}}=1-e^{k_{1} x \mp k_{1}^{2} t} .
$$

Substituting Eq. (39) into Eq. (1) and solving for $R_{1}$ and $R_{2}$ we find

$$
R_{1}=R_{2}=2
$$

This means that the single singular soliton solution is given by

$$
\begin{aligned}
& \left\{\begin{array}{l}
H(x, t)=-\frac{2 k_{1}^{2} \mathrm{e}^{k_{1} x+k_{1}^{2} t}}{\left(\mathrm{e}_{1}^{2} t-\mathrm{e}^{k_{1} x}\right)^{2}}, \\
u(x, t)=-\frac{2 k_{1} \mathrm{e}^{k_{1} x-k_{1}^{2} t}}{1-\mathrm{e}^{k_{1} x-k_{1}^{2} t}},
\end{array}\right. \\
& \left\{\begin{array}{l}
H(x, t)=-\frac{2 k_{1}^{2} \mathrm{e}^{k_{1} x+k_{1}^{2} t}}{\left(-1+\mathrm{e}^{k_{1} x+k_{1}^{2} t}\right)^{2}}, \\
u(x, t)=-\frac{2 k_{1} \mathrm{e}^{k_{1} x+k_{1}^{2} t}}{1-\mathrm{e}^{k_{1} x+k_{1}^{2} t}} .
\end{array}\right.
\end{aligned}
$$




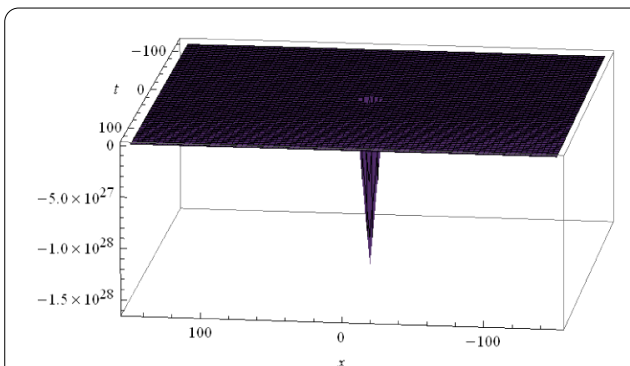

(a)

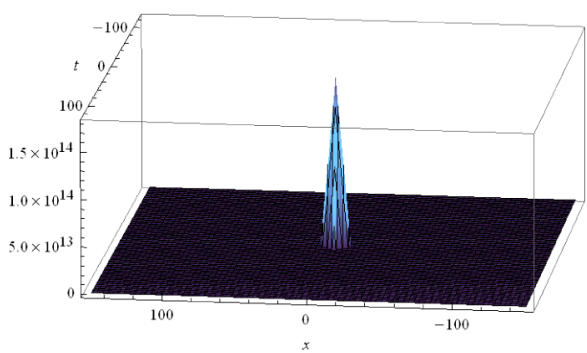

(b)

Figure 4 Plots of the solution described by Eq. (42). (a) The single singular soliton solution $H(x, t)$ for $k_{1}=0.4$. (b) The single singular soliton solution $u(x, t)$ for $k_{1}=0.4$.

Figure 4 shows the single singular soliton solution for Eq. (1) for some special values of the solution's parameters in Eq. (42).

For two singular soliton solutions, we set

$$
f(x, t)=1-\mathrm{e}^{\theta_{1}}-\mathrm{e}^{\theta_{2}}+a_{12} \mathrm{e}^{\theta_{1}+\theta_{2}}=1-\mathrm{e}^{k_{1} x \mp k_{1}^{2} t}-\mathrm{e}^{k_{2} x \mp k_{2}^{2} t}+a_{12} \mathrm{e}^{\left(k_{1}+k_{2}\right) x \mp\left(k_{1}^{2}+k_{2}^{2}\right) t} .
$$

Using Eq. (44) into Eq. (39) and substituting the result into Eq. (1), we obtain the phase shift

$$
a_{12}=0
$$

and hence we set

$$
a_{i j}=0, \quad 1 \leq i<j \leq N
$$

The two singular soliton solutions are obtained by substituting Eqs. (44) and (45) into Eq. (39), where we find

$$
\begin{aligned}
& \left\{\begin{array}{l}
H(x, t)=-\frac{2 \mathrm{e}^{\left(k_{1}^{2}+k_{2}^{2}\right) t}\left[k_{1}^{2} \mathrm{e}^{k_{1} x+k_{2}^{2} t}-\left(k_{1}-k_{2}\right)^{2} \mathrm{e}^{\left(k_{1}+k_{2}\right) x}+k_{2}^{2} \mathrm{e}^{k_{2} x+k_{1}^{2} t}\right]}{\left(-\mathrm{e}_{1}^{\left(k_{1}^{2}+k_{2}^{2}\right) t}+\mathrm{e}^{k_{1} x+k_{2}^{2} t}+\mathrm{e}^{k_{2} x+k_{1}^{2} t}\right)^{2}}, \\
u(x, t)=\frac{2\left(-k_{1} \mathrm{e}^{k_{1} x-k_{1}^{2} t}-k_{2} \mathrm{e}^{k_{2} x-k_{2}^{2} t}\right)}{1-\mathrm{e}^{k_{1} x-k_{1}^{2} t}-\mathrm{e}^{k_{2} x-k_{2}^{2} t}},
\end{array}\right. \\
& \left\{\begin{array}{l}
H(x, t)=-\frac{2 k_{1}^{2} \mathrm{e}^{k_{1} x+k_{1}^{2} t}-2 \mathrm{e}^{k_{2} x+k_{2}^{2} t}\left[\left(k_{1}-k_{2}\right)^{2} \mathrm{e}^{k_{1} x+k_{1}^{2} t}+k_{2}^{2}\right]}{\left(\mathrm{e}^{k_{1} x+k_{1}^{2} t}+\mathrm{k}^{k_{2} x+k_{2}^{2} t}\right)^{2}}, \\
u(x, t)=-\frac{2\left(k_{1} \mathrm{e}^{k_{1} x+k_{1}^{2} t}+\mathrm{k}_{2} \mathrm{e}^{k_{2} x+k_{2}^{2} t}\right)}{1-\mathrm{e}^{k_{1} x+k_{1}^{2} t}-\mathrm{e}^{k_{2} x+k_{2}^{2} t}} .
\end{array}\right.
\end{aligned}
$$

Figure 5 shows the two singular soliton solutions for Eq. (1) for some special values of the solution's parameters in Eq. (47).

For three singular soliton solutions, we set

$$
f(x, t)=1-e^{\theta_{1}}-e^{\theta_{2}}-e^{\theta_{3}}=1-e^{k_{1} x \mp k_{1}^{2} t}-e^{k_{2} x \mp k_{2}^{2} t}-e^{k_{3} x \mp k_{3}^{2} t} .
$$




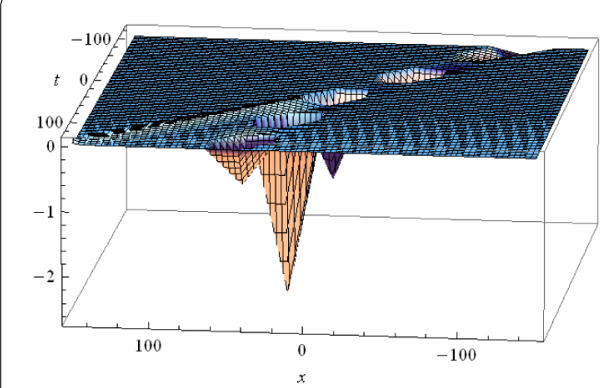

(a)

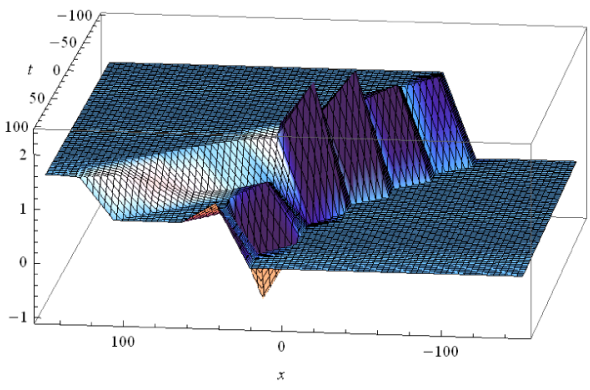

(b)

Figure 5 Plots of the solution described by Eq. (47). (a) The two singular soliton solutions $H(x, t)$ for $k_{1}=0.4, k_{2}=0.8$. (b) The two singular soliton solutions $u(x, t)$ for $k_{1}=0.4, k_{2}=0.8$.

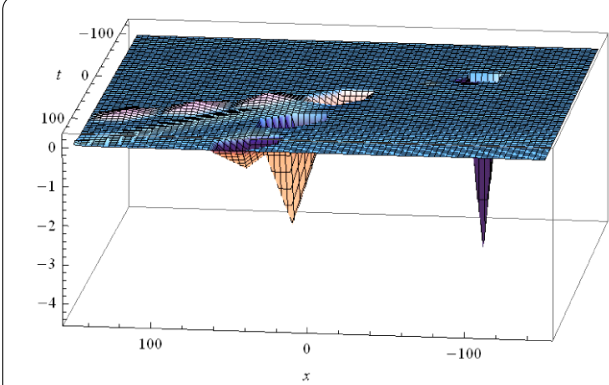

(a)

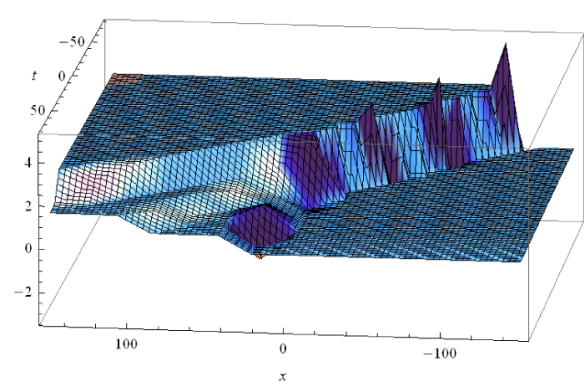

(b)

Figure 6 Plots of the solution described by Eq. (50). (a) The three singular soliton solutions $H(x, t)$ for $k_{1}=0.4, k_{2}=0.8, k_{3}=1.55$. (b) The three singular soliton solutions $u(x, t)$ for $k_{1}=0.4, k_{2}=0.8, k_{3}=1.45$.

Proceeding as before, we find the following three singular soliton solutions:

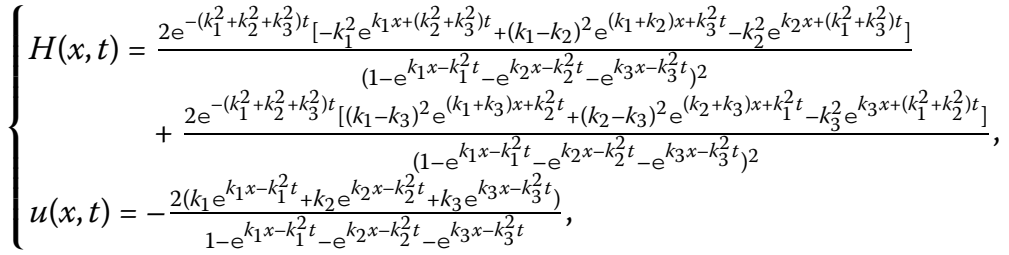

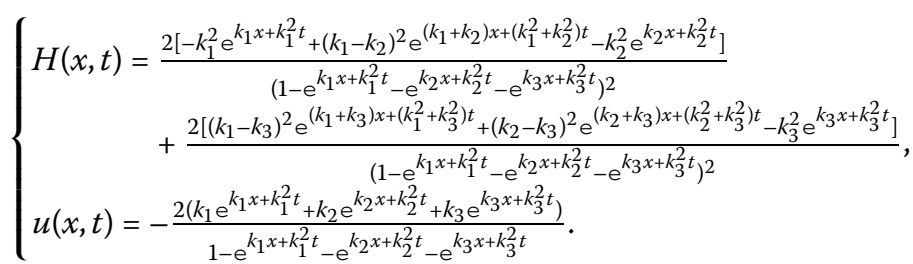

Figure 6 shows the three singular soliton solutions for Eq. (1) for some special values of the solution's parameters in Eq. (50).

Based on the last result, we can set the general singular soliton solutions as

$$
\left\{\begin{array}{l}
H(x, t)=\frac{2\left[-\sum_{i=1}^{N} k_{i}^{2} \mathrm{e}^{k_{i} x-k_{i}^{2} t}+\sum_{1 \leq i<j \leq N}\left(k_{i}-k_{j}\right)^{\left(k_{i}+k_{j}\right) x-\left(k_{i}^{2}+k_{j}^{2}\right) t}\right]}{u(x, t)=-\frac{2 \sum_{i=1}^{N} k_{i} \mathrm{e}^{k_{i} x-k_{i}^{2} t}}{1-\sum_{i=1}^{N} \mathrm{e}^{k_{i} x-k_{i}^{2} t}},},
\end{array}\right.
$$




$$
\left\{\begin{array}{l}
H(x, t)=\frac{2\left[-\sum_{i=1}^{N} k_{i}^{2} \mathrm{e}^{k_{i} x+k_{i}^{2} t}+\sum_{1 \leq i<j \leq N}\left(k_{i}-k_{j}\right)^{2} \mathrm{e}^{\left(k_{i}+k_{j}\right) x+\left(k_{i}^{2}+k_{j}^{2}\right) t}\right]}{\left(1-\sum_{i=1}^{N} \mathrm{e}^{k_{i} x+k_{i}^{2} t}\right)^{2}}, \\
u(x, t)=-\frac{2 \sum_{i=1}^{N} k_{i} \mathrm{e}^{k_{i} x+k_{i}^{2} t}}{1-\sum_{i=1}^{N} \mathrm{e}^{k_{i} x+k_{i}^{2} t}} .
\end{array}\right.
$$

\section{Conclusions}

The Hirota bilinear method is applied to emphasize the integrability of the variant Boussinesq equations. Multiple soliton solutions and multiple singular soliton solutions are formally derived. The analysis confirms the fact that the variant Boussinesq equations have $N$ soliton solutions, and have $N$ singular soliton solutions simultaneously. The results obtained for the phase shift $a_{i j}$ show that the system is resonance free. The method used here is standard and direct, so we believe that multiple soliton solutions and multiple singular soliton solutions may exist for other classes of nonlinear mathematic physics models, such as the coupled Kadomtsev-Petviashvili system, the Davey-Stewartson system, the generalized Hirota-Satsuma coupled KdV system, and so on. Further work on these aspects is worthy of performing.

\section{Competing interests}

The authors declare that they have no competing interests.

\section{Authors' contributions}

The authors have equal contributions to each part of this paper. All the authors read and approved the final manuscript.

\section{Author details}

'School of Mathematics and Physics, Lanzhou Jiaotong University, Lanzhou, 730070, P.R. China. ${ }^{2}$ School of Mechatronic Engineering, Lanzhou Jiaotong University, Lanzhou, 730070, P.R. China. ${ }^{3}$ Key Laboratory of Railway Vehicle Thermal Engineering, Lanzhou Jiaotong University, Ministry of Education, Lanzhou, 730070, P.R. China.

\section{Acknowledgements}

The authors would like to express their sincere thanks to editors and referees for their valuable suggestions and comments. This work was supported by the National Natural Science Foundation of China under Grant no. 11464027, the Scientific Research Foundation of the Higher Education Institutions of Gansu Province under Grant no. 2014A-053, and the Young Scholars Science Foundation of Lanzhou Jiaotong University under Grant no. 2013026.

Received: 28 September 2014 Accepted: 13 January 2015 Published online: 31 January 2015

\section{References}

1. Ablowitz, MJ, Clarkson, PA: Soliton, Nonlinear Evolution Equations and Inverse Scattering. Cambridge Universty Press, New York (1991)

2. Matveev, VB, Salle, MA: Darboux Transformation and Solitons. Springer, Berlin (1991)

3. Miura, MR: Backlund Transformation. Springer, Berlin (1978)

4. Hirota, R: Exact solution of the Korteweg-de Vries equation for multiple collisions of solitons. Phys. Rev. Lett. 27, 1192-1194 (1971)

5. Parkes, EJ, Duffy, BR: An automated tanh-function method for finding solitary wave solutions to non-linear evolution equations. Comput. Phys. Commun. 98, 288-300 (1996)

6. Fan, EG: Extended tanh-function method and its applications to nonlinear equations. Phys. Lett. A 277, 212-218 (2000)

7. Yan, CT: A simple transformation for nonlinear waves. Phys. Lett. A 224, 77-84 (1996)

8. Wang, ML: Exact solutions for a compound KdV-Burgers equation. Phys. Lett. A 213, 279-287 (1996)

9. Chun, C, Sakthivel, R: Homotopy perturbation technique for solving two-point boundary value problems-comparison with other methods. Comput. Phys. Commun. 181, 1021-1024 (2010)

10. Sakthivel, R, Chun, C, Lee, J: New travelling wave solutions of Burgers equation with finite transport memory. Z. Naturforsch. A 65, 633-640 (2010)

11. Abdou, MA: The extended F-expansion method and its application for a class of nonlinear evolution equations. Chaos Solitons Fractals 31, 95-104 (2007)

12. He, JH: Exp-function method for nonlinear wave equations. Chaos Solitons Fractals 30, $700-708$ (2006)

13. Sakthivel, R, Chun, C: New soliton solutions of Chaffee-Infante equations using the Exp-function method. Z. Naturforsch. A 65, 197-202 (2010)

14. Zayed, EME, Gepreel, KA: The $\left(G^{\prime} / G\right)$-expansion method for finding traveling wave solutions of nonlinear partial differential equations in mathematical physics. J. Math. Phys. 50, 013502 (2009)

15. Kim, H, Sakthivel, R: New exact traveling wave solutions of some nonlinear higher-dimensional physical models. Rep. Math. Phys. 70, 39-50 (2012) 
16. Kim, $\mathrm{H}, \mathrm{Bae}, \mathrm{JH}$, Sakthivel, R: Exact travelling wave solutions of two important nonlinear partial differential equations. Z. Naturforsch. A 69, 155-162 (2014)

17. Lou, $\mathrm{SY}, \mathrm{Ni}, \mathrm{GJ}$ : The relations among a special type of solitons in some $(D+1)$ dimensional nonlinear equations. J. Math. Phys. 30, 1614-1620 (1989)

18. Bai, $\mathrm{CJ}, \mathrm{ZhaO}, \mathrm{H}, \mathrm{Xu}, \mathrm{HY}$, Zhang, X: New traveling wave solutions for a class of nonlinear evolution equations. Int. J. Mod. Phys. B 25, 319-327 (2011)

19. Hirota, R, Ito, M: Resonance of solitons in one dimension. J. Phys. Soc. Jpn. 52, 744-748 (1983)

20. Hirota, R: The Direct Method in Soliton Theory. Cambridge University Press, Cambridge (2004)

21. Hirota, R: Exact solutions of the modified Korteweg-de Vries equation for multiple collisions of solitons. J. Phys. Soc. Jpn. 33, 1456-1458 (1972)

22. Hirota, R: Exact solutions of the Sine-Gordon equation for multiple collisions of solitons. J. Phys. Soc. Jpn. 33, 1459-1463 (1972)

23. Hietarinta, J: A search for bilinear equations passing Hirota's three-soliton condition. I. KdV-type bilinear equations. J. Math. Phys. 28, 1732-1742 (1987)

24. Hietarinta, J: A search for bilinear equations passing Hirota's three-soliton condition. II. mKdV-type bilinear equations. J. Math. Phys. 28, 2094-2101 (1987)

25. Wazwaz, AM: Partial Differential Equations and Solitary Waves Theory. Higher Education Press, Beijing (2009)

26. Sachs, RL: On the integrable variant of the Boussinesq system: Painlevé property, rational solutions, a related many-body system, and equivalence with the AKNS hierarchy. Physica D 30, 1-27 (1988)

27. Wang, ML: Solitary wave solutions for variant Boussinesq equations. Phys. Lett. A 199, 169-172 (1995)

28. Yan, ZY, Zhang, HQ: New explicit and exact travelling wave solutions for a system of variant Boussinesq equations in mathematical physics. Phys. Lett. A 252, 291-296 (1999)

29. Naz, R, Mahomed, FM, Hayat, T: Conservation laws for third-order variant Boussinesq system. Appl. Math. Lett. 23, 883-886 (2010)

30. Fan, EG, Hon, YC: A series of travelling wave solutions for two variant Boussinesq equations in shallow water waves. Chaos Solitons Fractals 15, 559-566 (2003)

31. Lü, DZ: Jacobi elliptic function solutions for two variant Boussinesq equations. Chaos Solitons Fractals 24, 1373-1385 (2005)

32. Yuan, $Y B, P u, D M, L i, S M$ : Bifurcations of travelling wave solutions in variant Boussinesq equations. Appl. Math. Mech. 27, 811-822 (2006)

33. Li, H, Ma, LL, Feng, DH: Single-peak solitary wave solutions for the variant Boussinesq equations. Pramana 80, 933-944 (2013)

\section{Submit your manuscript to a SpringerOpen ${ }^{\odot}$ journal and benefit from:}

- Convenient online submission

- Rigorous peer review

- Immediate publication on acceptance

- Open access: articles freely available online

- High visibility within the field

- Retaining the copyright to your article 